\title{
Piperlongumine attenuates collagen-induced arthritis via expansion of myeloid-derived suppressor cells and inhibition of the activation of fibroblast-like synoviocytes
}

\author{
JIAN SUN $^{1 *}$, PING XU ${ }^{2 *}$, XUEPING DU ${ }^{2}$, QINGGANG ZHANG ${ }^{1}$ and YUCHANG ZHU ${ }^{1}$ \\ ${ }^{1}$ Department of Orthopedics, Shanghai Tenth People's Hospital of Tongji University, Shanghai 200072; \\ ${ }^{2}$ Department of Orthopedics and Traumatology, Qidong Hospital of Traditional Chinese Medicine, \\ Nantong, Jiangsu 421600, P.R. China
}

Received March 30, 2014; Accepted August 6, 2014

DOI: $10.3892 / \mathrm{mmr} .2014 .3001$

\begin{abstract}
Piperlonguminine (PL), a key compound from the Piper longum fruit, is known to exhibit anti-tumor and anti-inflammatory activities. However, little is known about its effects on collagen-induced arthritis (CIA). Fibroblast-like synoviocytes (FLS) have a pivotal role in the development of rheumatoid arthritis (RA). Myeloid-derived suppressor cells (MDSCs) are able to suppress $\mathrm{T}$ cell responses and have important roles in the regulation of autoimmune arthritis. The current study investigated whether PL alters the progression of RA. It was determined that PL reduces the arthritis score and histopathologic lesions in a mouse model of CIA. PL also reduces the expression levels of serum anti-collagen II antibodies (anti-CII), tumor necrosis factor- $\alpha$ (TNF- $\alpha$ ), interleukin (IL)-1 $\beta$, IL-23 and IL-17 in CIA mice. In draining lymph nodes (DLNs), MDSCs were significantly expanded, however, the number of Th17 cells was markedly decreased by PL treatment. Additionally, PL reduced secretion of IL-1 $\beta$, IL-23 and IL-17 by TNF- $\alpha$-stimulated human RA FLS. PL significantly inhibited the migration and invasion of TNF- $\alpha$-stimulated human RA FLS. These results indicate that PL may be a candidate therapeutic agent for the treatment of RA, via the expansion of MDSCs and the inhibition of the Th17 response and activation of FLS.
\end{abstract}

Correspondence to: Dr Yuchang Zhu, Department of Orthopedics, Shanghai Tenth People's Hospital of Tongji University, 301 Yanchang Middle Road, Shanghai 200072, P.R. China

E-mail: yuchang_zhu@126.com

*Contributed equally

Key words: piperlongumine, rheumatoid arthritis, collagen-induced arthritis, myeloid-derived suppressor cells, fibroblast-like synoviocytes

\section{Introduction}

Rheumatoid arthritis (RA) is a chronic inflammatory autoimmune disease which primarily affects the synovia of joints. The synovium consists of a lining layer of fibroblast-like synoviocytes (FLS) that overlies the connective tissue of the synovial sublining (1). During the course of RA, massive leukocyte infiltration activates the FLS to produce proinflammatory mediators, including cytokines and chemokines $(2,3)$. Activated FLS become hyperplastic and form a pannus tissue that invades articular cartilage and bone $(3,4)$. Therefore, FLS perpetuates inflammation and destroys cartilage and bone.

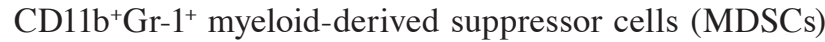
consist of a heterogeneous population of early myeloid progenitors, with potent immunosuppressive abilities (5). MDSCs were discovered in 1987 and previous studies have identified an important role for MDSCs in autoimmunity and inflammation (6-8). Expansion of MDSCs has been reported to reduce experimental autoimmune encephalomyelitis and the transfer of MDSCs has been demonstrated to reduce disease severity and significantly inhibit Th1 and Th17 responses (9-11). Notably, previous studies have implicated a role for MDSCs in RA. MDSCs potently suppress the expansion of autoreactive T cells, and inhibit collagen-induced arthritis (CIA) (12). It has been reported that an increase in circulating MDSCs negatively correlates with the levels of Th17 cells in patients with RA (13).

Piperlonguminine (PL), a major alkaloid isolated from Piper longum fruits, exhibits several biological activities, including anti-tumor and anti-inflammatory effects (14). However, the potential effect of PL on RA has not yet been investigated to the best of our knowledge. The aim of the present study was to investigate the effect of PL treatment on the migration of FLS and the progression of RA.

\section{Materials and methods}

Mice. Six to seven week-old male DBA/1 mice were bought from the Chinese Academy of Sciences (Shanghai, China) and kept under standard conditions (15) at the Tongji University School of Medicine (Shanghai, China). All experimental 
procedures were approved by the Institutional Animal Care and Use Committee of Tongji University.

Human patients with $R A$. Ten newly diagnosed patients with active RA who had not receive any treatment and fulfilled the 2010 American College of Rheumatology/European League Against Rheumatism criteria for RA (16) were recruited at the Shanghai Tenth People's Hospital of Tongji University (Shanghai, China). Patients with active disease were defined as those with a disease activity score in 28 joints of $>3.2$. This study was performed with the approval of the Ethics Committee of Tongji University in accordance with the Declaration of Helsinki. Informed consent was obtained from all patients.

Induction of CIA and PL treatment. CIA was induced in male DBA/1 mice via an intradermal injection of $100 \mu \mathrm{g}$ bovine type II collagen (CII; Chondrex, Inc., Redmond, WA, USA) emulsified with Complete Freund's adjuvant containing Mycobacterium tuberculosis (Chondrex, Inc.). Mice were boosted 21 days later with an injection $(100 \mu \mathrm{g}$ bovine type II collagen emulsified in Incomplete Freund's adjuvant (Chondrex, Redmond, WA, USA).

On day 28 following the first immunization, arthritis was observed in all mice and treatment with PL was initiated. Mice were randomly divided into two groups $(\mathrm{n}=8$ per group) and intraperitoneally injected with $2.4 \mathrm{mg} / \mathrm{kg} / \mathrm{day}$ PL (Sigma, St. Louis, MO, USA) or a vehicle control (dimethylsulfoxide; DMSO; Sigma, St. Louis, MO, USA) as described by Raj et al (17). PL treatment was continued until day 47.

Assessment of arthritis. Clinically, arthritic severity was characterized via the observation of joint properties and the inflammation of the surrounding tissue. The clinical arthritis was assessed every three days and scored as previously described (18). The maximal arthritis score per paw is 4 , and the maximal disease score per mouse is 16 .

Histopathologic analysis. The mouse joints were fixed in 4\% paraformaldehyde (Sigma), decalcified in EDTA (Sigma) and embedded in paraffin. Sections (thickness, $7 \mu \mathrm{m}$ ) were prepared and stained with hematoxylin and eosin (H\&E) and Safranin O (Promega Corporation, Madison, WI, USA to detect proteoglycans. On day 47 following immunization, the mice were sacrificed under $\mathrm{CO}_{2}$ anaesthesia and the hind paws were fixed in $10 \%$ buffered formalin, decalcified and embedded in paraffin. Joint sections (thickness, $6 \mu \mathrm{m}$ ) were prepared and stained with hematoxylin. Images were captured using an Olympus BX41 microscope (Olympus, Center Valley, PA, USA). The histological scores were evaluated, as previously described (18).

Measurement of anti-CII and serum cytokine levels. Serum concentrations of anti-CII IgG and IgG2a were measured using an enzyme-linked immunosorbent assay (ELISA). Blood was collected at the end of study, immediately following sacrifice. Microtiter plates were coated with CII $(10 \mu \mathrm{g} / \mathrm{ml})$, blocked and incubated with serially diluted test sera. Bound $\operatorname{IgG}$ was detected via incubation with horseradish peroxidase (HRP)-conjugated rat anti-mouse IgG or IgG2a, and tetramethylbenzidine substrate. Absorbance (wavelength, $450 \mathrm{~nm}$ ) was measured with an ELISA plate reader (3550; Bio-Rad, Hercules, CA, USA), and the values were represented in arbitrary optical density (OD) units.

Serum and supernatant TNF- $\alpha$, IL-1 $\beta$, IL-23 and IL-17 were measured using the BD Cytometric Bead Array (CBA) and specific ELISA kits for TNF- $\alpha$, IL-1 $\beta$, IL-23 and IL-17 (BD Biosciences, San Jose, CA, USA) according to the manufacturer's instructions. (BD Biosciences, San Jose, CA, USA) according to the manufacturer's instructions.

Flow cytometry. Draining lymph node (DLN) cells from each treatment group were cut and removed following sacrifice and made into a single cell suspension. The cells were washed, incubated with antibodies for fluorescence-activated cell sorting (FACS) and analyzed with a FACS Canto II flow cytometer. The following monoclonal antibodies were obtained from eBiosciences, Inc. (San Diego, CA, USA): Anti-CD11b, anti-Gr-1, anti-CD4 (phycoerythrin; PE), anti-CD25 (fluorescein isothiocyanate; FITC), anti-Foxp3 (PE-cyanine 5) and anti-IL-17 (FITC). Cells were restimulated with $50 \mathrm{ng} / \mathrm{ml}$ phorbol 12-myristate 13 -acetate and $500 \mathrm{ng} / \mathrm{ml}$ ionomycin in the presence of GolgiPlug ${ }^{\mathrm{TM}}$ for $5 \mathrm{~h}$ followed by intracellular staining. Cells were fixed, permeabilized and stained with fluorochrome-conjugated antibodies. The stained cells were then analyzed using a FACSCalibur or BD FACSAria instrument. Unless otherwise stated, all reagents and equipment was purchased from BD Biosciences.

Human RA FLS collection and culture. Synovial tissues were obtained from patients with RA during total knee replacement surgery or arthroscopic synovectomy. The FLS from five patients were treated with PL $(100 \mu \mathrm{g} / \mathrm{ml})$ or a vehicle. Tissues were digested with $4 \mathrm{mg} / \mathrm{ml}$ collagenase II (Sigma) in serum-free Dulbecco's modified Eagle's medium (DMEM; Gibco-BRL, Grand Island $<$ NY, USA) for $\geq 4 \mathrm{~h}$ at $37^{\circ} \mathrm{C}$. Cell suspensions were passed through a nylon mesh and the cell suspensions were collected via centrifugation at $500 \mathrm{x} \mathrm{g}$ for 5 min and re-suspended in DMEM supplemented with $10 \%$ fetal bovine serum (FBS; Gibco-BRL). Harvested cells were cultured in $75-\mathrm{cm}^{2}$ culture flasks (Costar, Cambridge, MA, USA) with DMEM supplemented with $10 \% \mathrm{FBS}$ at $37^{\circ} \mathrm{C}$ in $5 \% \mathrm{CO}_{2}$. Once the cells had grown to confluence they were detached with $0.25 \%$ trypsin, split at a ratio of $1: 3$ and re-cultured in DMEM under the same conditions. Cells obtained from the fourth to sixth passages were used in the following experiments.

Human RA FLS migration and invasion. In vitro migration assay of FLS chemotaxis was performed using the Boyden chamber method with an $8.0-\mu \mathrm{m}$ pore size (Transwell; Corning Inc., Corning, NY, USA). DMEM containing 10\% FBS as a chemoattractant was placed in the lower wells. FLS $\left(5 \times 10^{4}\right.$ cells $\left./ \mathrm{ml}\right)$ were suspended in serum-free DMEM in the upper wells. The chamber was incubated at $37^{\circ} \mathrm{C}$ under $5 \%$ $\mathrm{CO}_{2}$ for $6 \mathrm{~h}$. Following incubation, the non-migrating cells were removed from the upper surface of the filter using a cotton swab. The filters were fixed in methanol for $20 \mathrm{~min}$ and stained with $0.1 \%$ crystal violet for $20 \mathrm{~min}$. Chemotaxis was quantified by counting the stained cells which had migrated to 
A

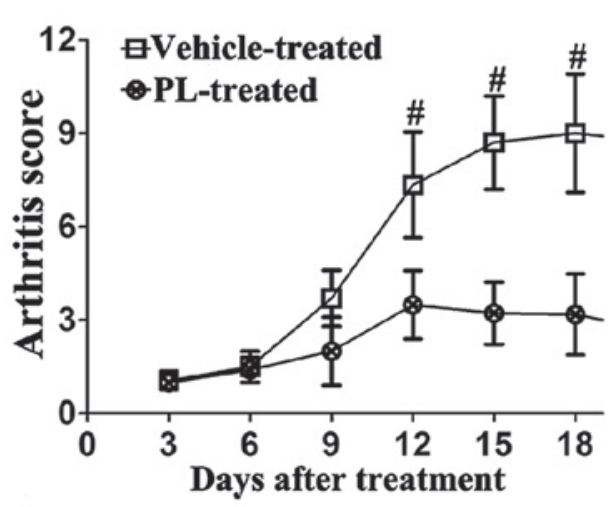

B

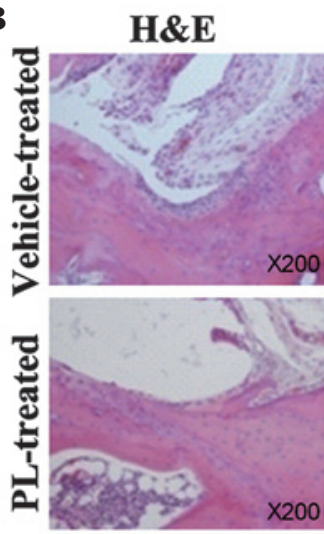

Safranin O

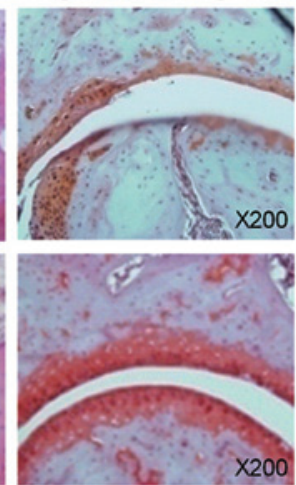

C
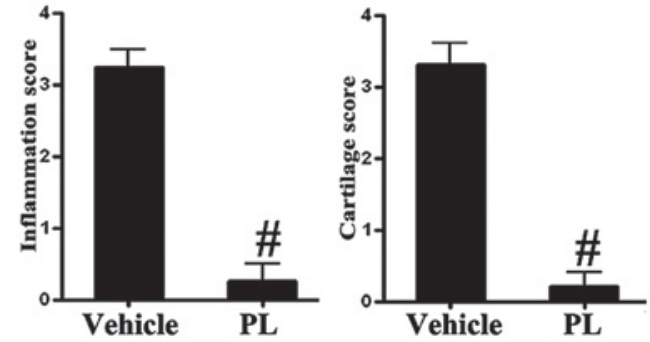

Figure 1. Piperlongumine (PL) reduced the levels of collagen-induced arthritis (CIA). The arthritis score was recorded every 3 days. Treatment with PL was initiated on day 28 following the first immunization, when clear arthritis was observed in all mice. (A) Arthritis score during the treatment. (B) Mouse CIA joints stained with hematoxylin and eosin (H\&E) and Safranin O. (C) Semi-quantitative histological analysis. ${ }^{~} \mathrm{P}<0.01$ vs. vehicle-treated control, $\mathrm{n}=8 \mathrm{mice}$ per group.

A

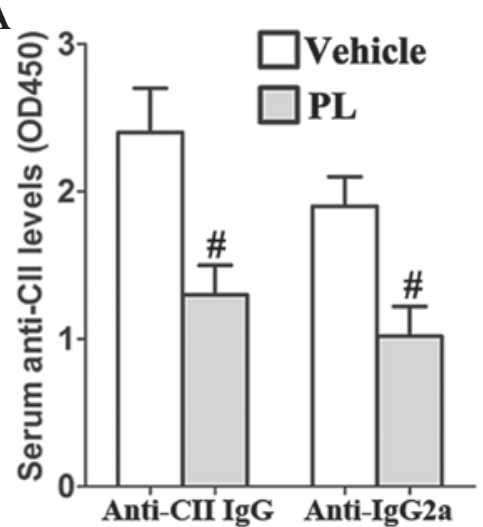

B

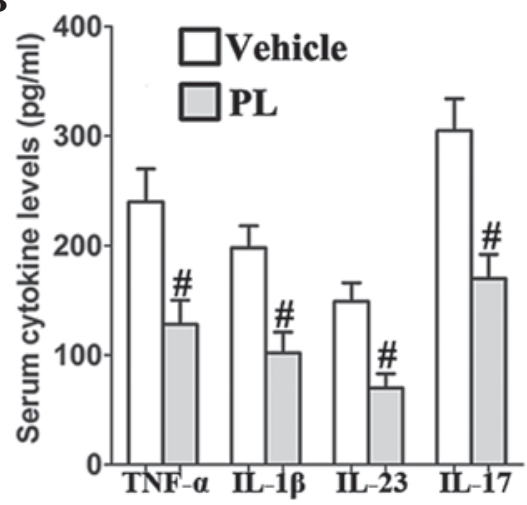

Figure 2. Piperlongumine (PL) reduced the serum anti-collagen II antibodies (anti-CII) and proinflammatory cytokines in collagen-induced arthritis mice. (A) Serum levels of anti-CII. (B) Serum levels of serum tumor necrosis factor- $\alpha$ (TNF- $\alpha$ ), interleukin (IL)-1 $\beta$, IL-23 and IL-17. " $\mathrm{P}<0.01$ vs. vehicle-treated control, $\mathrm{n}=8$ mice per group.

the lower side of the filter using an optical fluorescence microscope (System Microscope BX53; Olympus, Center Valley, PA, USA)

For the in vitro invasion assay, similar experiments were performed using inserts coated with a Matrigel basement membrane matrix (BD Biosciences, Oxford, UK). To investigate the effect of PL on RA FLS invasion, PL $(100 \mu \mathrm{g} / \mathrm{ml})$ was added to the upper and lower wells. Following treatment with TNF- $\alpha(100 \mathrm{ng} / \mathrm{ml})$, cells were observed under a fluorescence microscope (Olympus).

Statistical analysis. The differences between groups were determined by analysis of variance and comparison between two groups was analyzed with the Student's t-test using SPSS, version 16.0 (SPSS, Inc., Chicago, IL, USA). Data are presented as the mean \pm the standard error of the mean. $P<0.05$ was considered to indicate a statistically significant difference.

\section{Results}

PL reduces arthritis and histopathologic lesions in CIA mice. The clinical arthritis was assessed every three days and scored. PL was observed to attenuate the clinical score of CIA (Fig. 1A). In addition, H\&E and Safranin O staining were used to detect proteoglycans. It was revealed that the histopathologic damage, including inflammatory cell infiltration and cartilage 

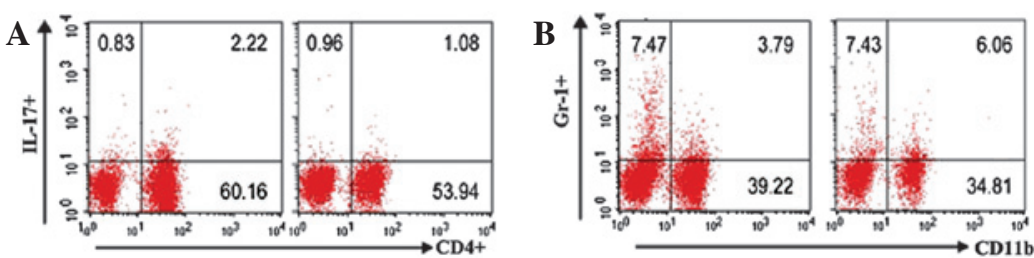

C

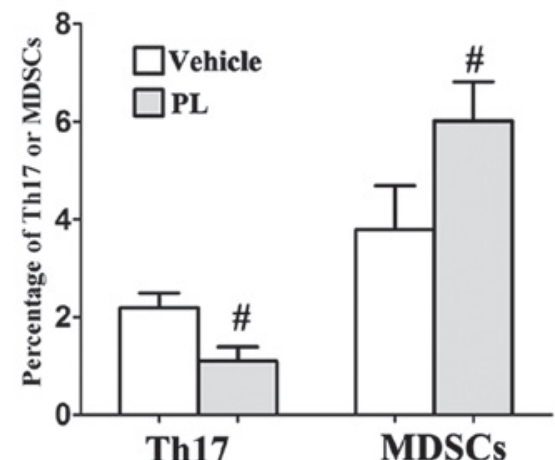

Figure 3. Piperlongumine (PL) markedly increased the number of $\mathrm{CD} 11 \mathrm{~b}^{+} \mathrm{Gr}-1^{+}$myeloid-derived suppressor cells (MDSCs) and decreased the number of $\mathrm{CD} 4^{+}$IL-1 $7^{+}$Th17 cells in the draining lymph nodes. Percentage of (A) Th17 cells and (B) MDSCs assessed by intracellular staining and flow cytometry.

(C) Quantitative analysis of Th17 cells and MDSCs. ${ }^{*} \mathrm{P}<0.01$ vs. vehicle-treated control, $\mathrm{n}=8$ mice per group.

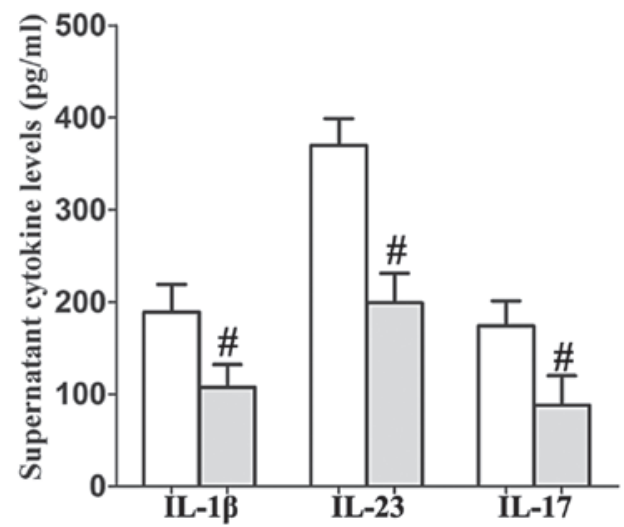

Figure 4. Piperlongumine (PL) reduced secretion of interleukin (IL)-1 $\beta$, IL-23 and IL-17 by tumor necrosis factor- $\alpha$ stimulated human rheumatoid arthritis fibroblast-like synoviocytes. ${ }^{*} \mathrm{P}<0.01$ vs. vehicle-treated control, $\mathrm{n}=5$ patients per group.
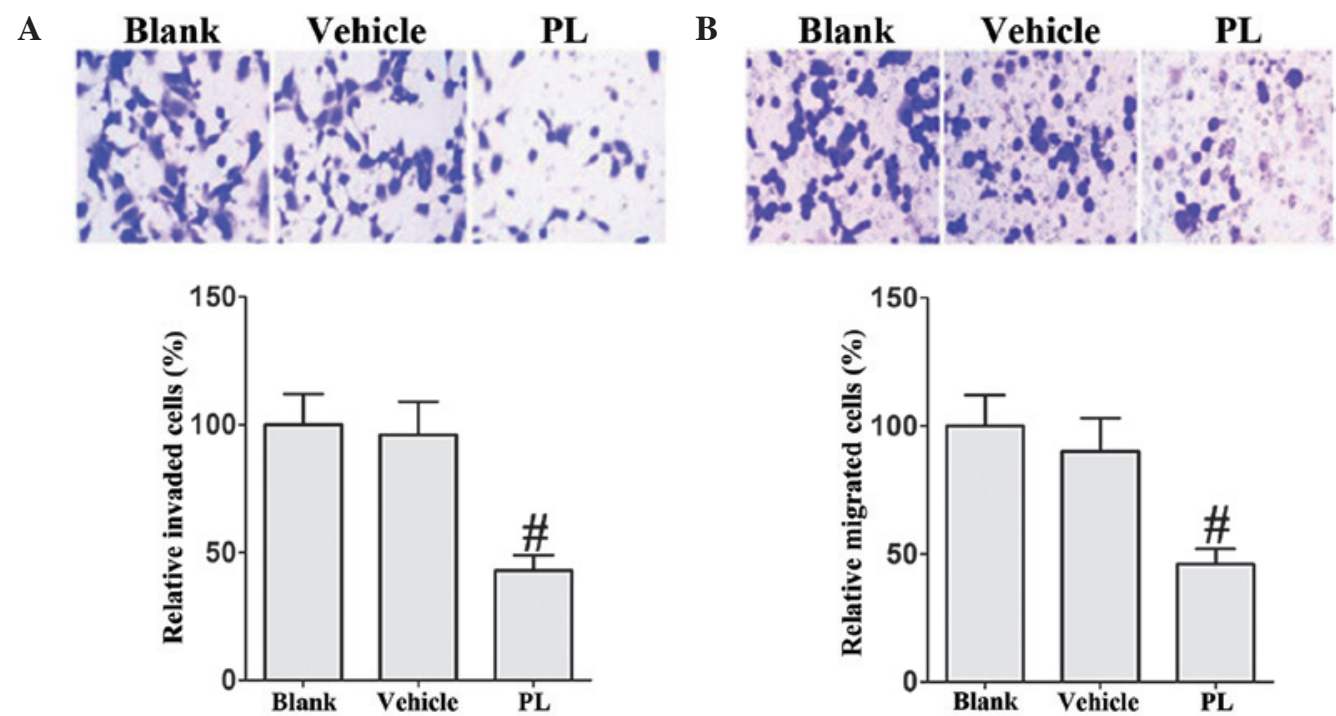

Figure 5. Piperlongumine inhibited the migration and invasion of rheumatoid arthritis fibroblast-like synoviocytes (RA FLS). (A) Piperlongumine significantly inhibited the IL-1 $\beta$-induced (100 ng/ml) migratory behavior of RA FLS. (B) Piperlongumine significantly reduced the IL-1 $\beta$-induced (100 ng/ml) invasion of RA FLS. ${ }^{\#} \mathrm{P}<0.01$, vs. vehicle-treated control, $\mathrm{n}=5$ patients per group. 
destruction, was significantly attenuated in PL-treated CIA mice in comparison with that in the vehicle-treated CIA control mice (Fig. 1B and C).

$P L$ reduces the levels of serum anti-CII and proinflammatory cytokines in CIA mice. PL significantly reduced the serum anti-CII IgG and IgG2a levels as compared with those of the vehicle-treated CIA control mice (Fig. 2A). In addition, the results revealed that $\mathrm{PL}$ inhibits the production of serum TNF- $\alpha$, IL-1 $\beta$, IL-23 and IL-17 in CIA mice as compared with that in the vehicle-treated CIA control mice (Fig. 2B).

PL increases the levels of MDSCs and decreases the levels of Th17. To evaluate the effect of PL on immune cell responses in vivo, the frequencies of MDSCs, $\mathrm{T}_{\text {regs }}$ and Th17 cells were examined in the DLNs of the control and PL-treated CIA mice. The percentage of Th17 cells was markedly decreased in the DLNs of the PL-treated CIA mice compared with that of the vehicle-treated CIA control mice (Fig. 3A and C). Conversely, the number of $\mathrm{CD} 11 \mathrm{~b}^{+} \mathrm{Gr}-1^{+} \mathrm{MDSC}$ showed a marked increase in the DLN of the PL-treated CIA mice compared with that of the vehicle-treated CIA control mice (Fig. 3B and C). In addition, the $\mathrm{T}_{\text {regs }} \mathrm{CD}^{+}, \mathrm{CD} 25^{+}$and Foxp $3^{+}$frequency were not affected by PL treatment $(9.8 \pm 1.27$, vs. $10.1 \pm 1.31 \%$; $P>0.05)$.

$P L$ reduces the secretion of $I L-1 \beta, I L-23$ and $I L-17$ by TNF- $\alpha$-stimulated human RA FLS. To determine whether PL affects human RA FLS in a similar way to that observed in mice, the human RA FLS were pretreated with PL for $24 \mathrm{~h}$ and then stimulated by human TNF- $\alpha$ (100 $\mathrm{ng} / \mathrm{ml})$ for $48 \mathrm{~h}$. The supernatants were then collected. It was revealed that PL reduced the levels of secretion of IL-1 $\beta$, IL-23 and IL-17 by human RA FLS as detected in the supernatants compared with those in the controls (Fig. 4).

$P L$ restricts the migration and invasion of human $R A F L S$. Subsequently, the effect of PL on migration and invasion of human RA FLS was investigated. Treatment with PL substantially inhibited the migration and invasion of TNF- $\alpha$-induced RA FLS compared with that of the vehicle controls (Fig. 5). These results indicate that PL inhibits the migratory behavior of human RA FLS.

\section{Discussion}

The results of the present study demonstrated the effects of PL in a mouse model of CIA and in human RA FLS. PL effectively alleviated CIA in mice, as indicated by the notable reductions in the clinical arthritis score and the histological lesions of CIA. The therapeutic effects of PL may be due to immunomodulatory and anti-inflammatory activities. Immunomodulatory activity was observed via the enhancement of MDSCs and the suppression of the Th17 response. Anti-inflammatory activity was demonstrated through the reduction of systemic levels of pro-inflammatory cytokines. These therapeutic effects of $\mathrm{PL}$ are also mediated by a decrease in migratory behavior as demonstrated in human RA FLS.

Although the etiology of RA remains unknown and controversy persists concerning the role of humoral and cellular autoimmunity in the pathogenesis of $\mathrm{RA}, \mathrm{CD} 4^{+} \mathrm{T}$ cells and pro-inflammatory cytokines have been shown to have an important role in CIA $(19,20)$. MDSCs are of myeloid origin and are able to suppress $\mathrm{T}$ cell responses. The roles of MDSCs in autoimmune diseases have recently been elucidated in autoimmune arthritis. In a mouse model of CIA, MDSCs inhibited the proliferation of $\mathrm{CD}^{+} \mathrm{T}$ cells and their differentiation into Th17 cells in addition to inhibiting the production of IFN- $\gamma$, IL- 2 , TNF- $\alpha$, and IL- 6 by CD4 $4^{+}$T cells in vitro, while promoting the production of IL-10 (19). Adoptive transfer of MDSCs reduced the severity of CIA in vivo, which was accompanied by a decrease in the number of $\mathrm{CD}^{+} \mathrm{T}$ cells and Th17 cells in the DLNs (21). A subsequent study indicated that the transfer of MDSCs significantly reduces the clinical score of arthritis and alleviates joint inflammation and the progression of CIA in antigen-induced arthritis and CIA models via the inhibition of the proinflammatory immune response of $\mathrm{CD}^{+} \mathrm{T}$ cells (22). These observations indicate that MDSCs have crucial roles in the regulation of autoimmune arthritis. In addition, Th17 cells are of critical importance in immune response. Th17 cells produce cytokines with pro-inflammatory effects, including IL-17, IL-6, IL-21, IL-22 and TNF- $\alpha$, all of which have a role in the immunopathogenesis of RA (23). IL-23 is involved in the maintenance of Th17 cells and the IL-23/Th17 axis has been demonstrated to be a potential target for the treatment of human RA (24). In the current study, it was determined that PL treatment inhibits the production of serum anti-CII, TNF- $\alpha$, IL-1 $\beta$, IL-23 and IL-17 in CIA mice. Additionally, PL treatment reduces the secretion of IL-1 $\beta$, IL-23 and IL-17 by TNF- $\alpha$-stimulated human RA FLS.

Notably, FLS are one of the dominant types of cell in the hyperplastic rheumatoid synovium and they have an important role in the pathogenesis of RA (25). A previous study indicated that the capacity of FLS to migrate from joint to joint, and the mechanisms by which FLS mediate persistence may offer disease-specific therapeutic targets in human RA (26). In the present study, it was revealed that PL significantly inhibits the TNF- $\alpha$-induced migration and invasion of RA FLS.

In conclusion, the results of the current study revealed that $\mathrm{PL}$ reduces the arthritis score and joint destruction via the expansion of MDSCs and the inhibition of the activation of FLS. Hence, it is hypothesized that PL may be a candidate therapeutic agent for RA.

\section{References}

1. Jang E, Kim HR, Cho SH, Paik DJ, Kim JM, Lee SK and Youn J: Prevention of spontaneous arthritis by inhibiting homeostatic expansion of autoreactive $\mathrm{CD} 4^{+} \mathrm{T}$ cells in the $\mathrm{K} / \mathrm{BxN}$ mouse model. Arthritis Rheum 54: 492-498, 2006.

2. Stanford SM, Maestre MF, Campbell AM, Bartok B, Kiosses WB, Boyle DL, Arnett HA, Mustelin T, Firestein GS and Bottini N: Protein tyrosine phosphatase expression profile of rheumatoid arthritis fibroblast-like synoviocytes: a novel role of $\mathrm{SH} 2$ domain-containing phosphatase 2 as a modulator of invasion and survival. Arthritis Rheum 65: 1171-1180, 2013.

3. Chang SK, Gu Z and Brenner MB: Fibroblast-like synoviocytes in inflammatory arthritis pathology: the emerging role of cadherin-11. Immunol Rev 233: 256-266, 2010.

4. Lee HS, Ka SO, Lee SM, Lee SI, Park JW and Park BH: Overexpression of sirtuin 6 suppresses inflammatory responses and bone destruction in mice with collagen-induced arthritis Arthritis Rheum 65: 1776-1785, 2013.

5. Goh C, Narayanan S and Hahn YS: Myeloid-derived suppressor cells: the dark knight or the joker in viral infections? Immunol Rev 255: 210-221, 2013. 
6. Young MR, Newby M and Wepsic HT: Hematopoiesis and suppressor bone marrow cells in mice bearing large metastatic Lewis lung carcinoma tumors. Cancer Res 47: 100-105, 1987.

7. Monu NR and Frey AB: Myeloid-derived suppressor cells and anti-tumor T cells: a complex relationship. Immunol Invest 41 : 595-613, 2012.

8. Kong YY, Fuchsberger M, Xiang SD, Apostolopoulos V and Plebanski M: Myeloid derived suppressor cells and their role in diseases. Curr Med Chem 20: 1437-1444, 2013.

9. Ioannou M, Alissafi T, Boon L, Boumpas D and Verginis P. In vivo ablation of plasmacytoid dendritic cells inhibits autoimmunity through expansion of myeloid-derived suppressor cells. J Immunol 190: 2631-2640, 2013.

10. Alabanza LM, Esmon NL, Esmon CT and Bynoe MS: Inhibition of endogenous activated protein $\mathrm{C}$ attenuates experimental autoimmune encephalomyelitis by inducing myeloid-derived suppressor cells. J Immunol 191: 3764-3777, 2013.

11. Ioannou M, Alissafi T, Lazaridis I, Deraos G, Matsoukas J, Gravanis A, Mastorodemos V, Plaitakis A, Sharpe A, Boumpas D and Verginis P: Crucial role of granulocytic myeloid-derived suppressor cells in the regulation of central nervous system autoimmune disease. J Immunol 188: 1136-1146, 2012.

12. Egelston C, Kurkó J, Besenyei T, Tryniszewska B, Rauch TA, Glant TT and Mikecz K: Suppression of dendritic cell maturation and $\mathrm{T}$ cell proliferation by synovial fluid myeloid cells from mice with autoimmune arthritis. Arthritis Rheum 64: 3179-3188, 2012.

13. Jiao Z, Hua S, Wang W, Wang H, Gao J and Wang X: Increased circulating myeloid-derived suppressor cells correlated negatively with Th17 cells in patients with rheumatoid arthritis Scand J heumatol 42: 85-90, 2013.

14. Ku SK, Kim JA and Bae JS: Piperlonguminine downregulates endothelial protein $\mathrm{C}$ receptor shedding in vitro and in vivo. Inflammation 37: 435-442, 2014.

15. Khathi A, Masola B and Musabayane CT: Effects of Syzygium aromaticum-derived oleanolic acid on glucose transport and glycogen synthesis in the rat small intestine. J Diabetes 5: 80-87, 2013.
16. Nakagomi D, Ikeda K, Okubo, et al: Ultrasound can improve the accuracy of the 2010 American College of Rheumatology/European League Against Rheumatism classification criteria for rheumatoid arthritis to predict the requirement for methotrexate treatment. Arthritis Rheum 65: 890-898, 2013.

17. Raj L, Ide T, Gurkar AU, Foley M, Schenone M, Li X, et al: Selective killing of cancer cells by a small molecule targeting the stress response to ROS. Nature 475: 231-234, 2011.

18. Mauri C, Mars LT, and Londei M: Therapeutic activity of agonistic monoclonal antibodies against CD40 in a chronic autoimmune inflammatory process. Nat Med 6: 673-679, 2000.

19. Kotzin B and Kappler J: Targeting the T cell receptor in rheumatoid arthritis. Arthritis Rheum 41: 1906-1910, 1998.

20. Malemud CJ and Miller AH: Pro-inflammatory cytokine-induced SAPK/MAPK and JAK/STAT inrheumatoid arthritis and the new anti-depression drugs. Expert Opin Ther Targets 12: 171-183, 2008.

21. Fujii W, Ashihara E, Hirai H, Nagahara H, Kajitani N, Fujioka K, Murakami K, Seno T, Yamamoto A, Ishino H, Kohno M, Maekawa T and Kawahito Y: Myeloid-derived suppressor cells play crucial roles in the regulation of mouse collagen-induced arthritis. J Immunol 191: 1073-1081, 2013.

22. Zhang L, Zhang Z, Zhang H, Wu M and Wang Y: Myeloid-derived suppressor cells protect mouse models from autoimmune arthritis via controlling inflammatory response. Inflammation 37: 670-677, 2014.

23. Noack M and Miossec P: Th17 and regulatory T cell balance in autoimmune and inflammatory diseases. Autoimmun Rev 13: 668-677, 2014.

24. Miossec P and Kolls JK: Targeting IL-17 and TH17 cells in chronic inflammation. Nat Rev Drug Discov 11: 763-776, 2012.

25. Neumann E, Lefèvre S, Zimmermann B, Gay S and Müller-Ladner U: Rheumatoid arthritis progression mediated by activated synovial fibroblasts. Trends Mol Med 16: 458-468, 2010.

26. Filer A: The fibroblast as a therapeutic target in rheumatoid arthritis. Curr Opin Pharmacol 13: 413-419, 2013. 